\title{
Unbalanced interval-valued OWA operators
}

\author{
Laura De Miguel • Humberto Bustince • \\ Edurne Barrenechea - Miguel Pagola . \\ Javier Fernandez
}

Received: date / Accepted: date

\begin{abstract}
In this work, we introduce a new class of functions defined on the interval-valued setting. These functions extend classical OWA operators but allow for different weighting vectors to handle the lower bounds and the upper bounds of the considered intervals. As a consequence, the resulting functions need not be an interval-valued aggregation function, so we study, in the case of the lexicographical order, when these operators give an interval as output and are monotone. We also discuss an illustrative example on a decision making problem in order to show the usefulness of our developments.
\end{abstract}

Keywords Interval-valued OWA operator · Admissible order · Aggregation functions · Decision making

\section{Introduction}

Aggregation functions $[2,3,9,12]$ are crucial tools for most artificial science and computer science applications whenever information which comes from different (homogeneous or heterogeneous) sources must be fused. These functions are just required to satisfy appropriate boundary and monotonicity conditions. These are quite natural conditions when we are dealing with real numbers, e.g., with data in the unit hypercube.

However, things are a bit more complicate when we have to deal with data which are not real numbers [13], specially if no natural linear order exists for

L. De Miguel, H. Bustince, E. Barrenechea, M. Pagola, J. Fernandez

Departamento Automática y Computación, Universidad Publica de Navarra, Campus Arrosadia s/n, 31006 Pamplona, Spain

Tel.: +34-94-8169254

Fax: +34-94-8168924

E-mail: laura.demiguel,bustince,edurne.barrenechea,miguel.pagola,fcojavier.fernandez@unavarra.es

L. De Miguel, H. Bustince, E. Barrenechea, M. Pagola, J. Fernandez

Institute of Smart Cities, Universidad Publica de Navarra, Campus Arrosadia s/n, 31006

Pamplona, Spain 
such data [8]. This is the case, for instance, if we must work with intervals, as it happens when it exists uncertainty or lack of knowledge around the information to be handled $[1,4,16]$. If, for the sake of simplicity, we restrict ourselves to closed subintervals of the unit interval, it is straight to define a partial order: it is enough to consider the order which is inherited from the one of real numbers. However, this order is not linear, that is, there exist pairs of intervals which are not comparable in terms of this relation.

The problem worsens if we consider the extension of some specific types of aggregation operators to interval-valued setting, as it is the case, for instance, of ordered weighted aggregation (OWA) operators [5] or, more generally, of Choquet integrals $[10,17]$. These types of operators require, as a first step to compute them, that the inputs are ordered. So, if we want to apply it to any $n$-tuple of intervals, we must find a linear order which allows us to compare any pair of intervals.

This problem was considered in [6] (see also [14]), where the notion of admissible order was introduced. An admissible order for intervals is a linear order which extends the partial order induced by the classical order relationship in $\mathbb{R}$. These admissible orders can be defined in terms of real-valued aggregation functions which fulfill appropriate conditions, and they include most of the linear orders between intervals that have been considered in the literature, as the lexicographical ones or the order defined by Xu and Yager in [18] in terms of score and accuracy functions.

Once admissible orders have been defined, it is straight to provide a definition of aggregation function where the monotonicity condition is defined with respect to the considered admissible order. In this sense, in [7] the problem of defining Choquet integrals and OWA operators for interval-valued data was considered.

In this work, we go one step further from the developments in [7]. Our objective is to define the so-called Unbalanced Interval-Valued OWA (UIVOWA) operators in terms of two different weighting vectors, one for the lower bounds and another one for the upper bounds.

The UIVOWA operator, however, needs not be an interval-valued aggregation function, since, for instance, the output may not be an interval. For this reason, we study which conditions allow us to ensure that the result is an interval. We do not do this for the general case of admissible orders, but just for the specific case of the lexicographical orders, since the whole analysis would be too long for this paper.

The usefulness of our developments is clear for those applications where OWA operators have shown themselves very fruitful, as it is the case of decisionmaking problems $[7,11,19]$. In this sense, we also discuss in this work an illustrative example on a simplified problem to see how our theory can be applied in this framework.

The structure of this work is as follows. In the next section we recall several preliminary definitions and results. In Section 3 we introduce the main concept of Unbalanced Interval-Valued OWA operator and we discuss, in the 
case of lexicographical orders, when we recover an interval-valued aggregation. In Section 4 we present an illustrative example in decision-making.

\section{Preliminaries}

In this section we recall several notions and definitions which are necessary for our subsequent developments. Furthermore, we also fix some notations for the rest of the paper.

We denote by $L([0,1])$ the set of closed subintervals of the unit interval, i.e.,

$$
L([0,1])=\{\mathrm{x}=[\underline{X}, \bar{X}]) \mid 0 \leq \underline{X} \leq \bar{X} \leq 1\} .
$$

In particular, we denote $0_{L}=[0,0]$ and $1_{L}=[1,1]$.

The usual order in $L\left([0,1]\right.$ is that inherited from $\mathbb{R}^{2}$, namely:

$$
[\underline{X}, \bar{X}] \preceq_{2}\left[\underline{Y}, q_{2} \bar{Y}\right] \text { if and only if } \underline{X} \leq \underline{Y} \text { and } \bar{X} \leq \bar{Y} \text {. }
$$

However, for many applications it is necessary to have the possibility of comparing any two data. This consideration leads to the notion of admissible order[7].

Definition 1 Let $\leq_{L}$ be an order in $L([0,1])$. The relation $\leq$ is an admissible order if

1. it is linear (i.e., for every $\mathbf{x}, \mathbf{y} \in L([0,1])$ it holds that $\mathbf{x} \leq_{L} \mathbf{y}$ or $\left.\mathbf{y} \leq_{L} \mathbf{x}\right)$, and

2. for all $\boldsymbol{x}, \boldsymbol{y} \in L([0,1])$, such that $\boldsymbol{x} \preceq_{2} \boldsymbol{y}$ it holds that $\boldsymbol{x} \leq_{L} \boldsymbol{y}$.

Remark 1 Note that for every admissible order $\leq_{L}$ and for every $\mathbf{x} \in L([0,1])$ it holds that $0_{L} \leq_{L} \mathbf{x} \leq_{L} 1_{L}$. That is, $0_{L}$ and $1_{L}$ are the top and the bottom elements, respectively, in $\left(L([0,1]), \leq_{L}\right)$, whatever the admissible order $\leq_{L}$ is.

That is, an admissible order is a linear order which extends the usual partial order between intervals.

Example 1 The three more relevant examples of admissible orders that can be found in the literature are the following.

1. Lexicographical order with respect to the first variable. $\mathbf{x} \leq_{\text {lex } 1} \mathbf{y}$ if $\underline{X}<\underline{Y}$ or $\underline{X}=\underline{Y}$ and $\bar{X} \leq \bar{Y}$.

2. Lexicographical order with respect to the second variable. $\mathbf{x} \leq_{\text {lex } 2} \mathbf{y}$ if $\bar{X}<$ $\bar{Y}$ or $\bar{X}=\bar{Y}$ and $X \leq Y$.

3. $X u$ and Yager's order [18]:

$$
[\underline{X}, \bar{X}] \leq_{X Y}[\underline{Y}, \bar{Y}] \text { if }\left\{\begin{array}{l}
\underline{X}+\bar{X}<\underline{Y}+\bar{Y} \text { or } \\
\underline{X}+\bar{X}=\underline{Y}+\bar{Y} \text { and } \bar{X}-\underline{X} \leq \bar{Y}-\underline{Y} .
\end{array}\right.
$$


Remark $2 X u$ and Yager's order was initially introduced in the Atanassov intuitionistic setting. However, as it is well known, it exists an straight mathematical equivalence between Atanassov intuitionistic fuzzy sets and intervalvalued fuzzy sets. Nevertheless, the order considered here is not exactly the same which was originally defined by its authors, since the second inequality is reversed here.

Another key notion which is at the basis of our present work is that of aggregation function. The definition of aggregation in the unit interval is widely known. Nevertheless, we recall the definition here.

Definition 2 An aggregation function is a function $M:[0,1]^{n} \rightarrow[0,1]$ such that

1. $M(0, \ldots, 0)=0$ and $M(1, \ldots, 1)=1$;

2. $M$ is increasing in each variable.

A particular instance of aggregation functions frequently used in many application are OWA operators given by Yager $[3,20]$.

Definition 3 Let $w$ be a weighting vector, i.e, $w=\left(w_{1}, \ldots, w_{n}\right) \in[0,1]^{n}$ with $w_{1}+\ldots+w_{n}=1$. The Ordered Weighted Aggregation operator associated with $w, O W A_{w}$, is a mapping $O W A_{w}:[0,1]^{n} \longrightarrow[0,1]$ defined by

$$
O W A_{w}\left(x_{1}, \ldots, x_{n}\right)=\sum_{i=1}^{n} w_{i} x_{(i)}
$$

where $x_{(i)}, i=1, \ldots, n$, denotes the $i-$ th greatest component of the input $\left(x_{1}, \ldots, x_{n}\right)$.

Aggregation functions can be used to define admissible orders in $L([0,1])$ as follows.

Proposition 1 Let $M_{1}, M_{2}$ be two aggregation functions $M_{1}, M_{2}:[0,1]^{2} \rightarrow$ $[0,1]$ such that for all $(a, b),(c, d) \in[0,1]^{2}$ with $a \leq b$ and $c \leq d$ the equalities $M_{1}(a, b)=M_{1}(c, d) M_{2}(a, b)=M_{2}(c, d)$ hold simultaneously if and only if $a=c$ y $b=d$. if

Then the relation $\leq_{M_{1}, M_{2}}$ on $L([0,1])$ given by $[a, b] \leq_{M}[c, d]$ if and only

i) $M_{1}(a, b)<M_{1}(c, d)$ or

ii) $M_{1}(a, b)=M_{1}(c, d)$ and $M_{2}(a, b) \leq M_{2}(c, d)$

is an admissible order on $L([0,1])$.

Example 2 1. The lexicographical order with respect to the first variable is recovered taking $M_{1}(x, y)=x$ and $M_{2}(x, y)=y$.

2. The lexicographical order with respect to the second variable is recovered taking $M_{1}(x, y)=y$ and $M_{2}(x, y)=x$.

3. $X u$ and Yager's order is recovered taking $M_{1}(x, y)=\frac{x+y}{2}$ and $M_{2}(x, y)=y$. 
Among admissible orders one very important class is generated by means of the $K_{\alpha}$ operators.

Definition 4 Let $\alpha \in[0,1]$. The operator $K_{\alpha}: L([0,1]) \rightarrow[0,1]$ is defined by:

$$
K_{\alpha}([\underline{X}, \bar{X}]=(1-\alpha) \underline{X}+\alpha \bar{X} .
$$

Note that $K_{0}$ corresponds to the projection with respect to the first component and $K_{1}$ corresponds to the projection with respect to the second component. In general, we have the following result:

Proposition 2 Let $\alpha_{1}, \alpha_{2} \in[0,1]$, with $\alpha_{1} \neq \alpha_{2}$. then, the order $\leq_{\alpha_{1}, \alpha_{2}}$ defined as in Proposition 1 with $M_{1}(x, y)=\left(1-\alpha_{1}\right) x+\alpha_{1} y$ and $M_{2}(x, y)=$ $\left(1-\alpha_{2}\right) x+\alpha_{1} 2$ is an admissible order.

Remark 3 The lexicographical order with respect to the first component is the same as $\leq_{0,1}$ whereas the lexicographical order with respect to the second component corresponds to $\leq_{1,0}$ and Xu and Yager's order corresponds to $\leq_{\frac{1}{2}, 1}$.

The definition of aggregation function can be extended to $L([0,1]$ as follows.

Definition 5 Let $\leq_{L}$ be an order in $L([0,1])$. An aggregation function $M$ on $L([0,1])$ with respect to the order $\leq_{L}$ is a mapping $M:(L([0,1]))^{n} \rightarrow \leq$ $L([0,1])$ satisfying:

1. $M\left(0_{L}, \ldots, 0_{L}\right)=0_{L}, \quad M\left(1_{L}, \ldots, 1_{L}\right)=1_{L}$,

2. $M\left(\mathbf{x}_{1}, \ldots, \mathbf{x}_{\mathbf{n}}\right) \leq_{L} M\left(\mathbf{y}_{\mathbf{1}}, \ldots, \mathbf{y}_{\mathbf{n}}\right)$ whenever $\mathbf{x}_{\mathbf{i}} \leq_{L} \mathbf{y}_{\mathbf{i}}$ for every $i \in\{1, \ldots, n\}$.

Finally, we also recall here the definition of interval-valued fuzzy set $[15,8]$.

Definition 6 An interval-valued fuzzy set $A$ over the universe $X \neq \emptyset$ is defined as:

$$
A=\left\{\left(x, \mu_{A}(x)\right) \mid x \in X\right\} .
$$

where $\mu_{A}: X \rightarrow L([0,1]$ is the membership function of the set $A$.

We denote by $I V F S(X)$ the set of all interval-valued fuzzy sets on the universe $X$. Every order $\leq_{L}$ in $L([0,1])$ induces a (partial) order in $I V F S(X)$ , (that we denote by $\leq_{L}$, too), given by:

$$
A \leq_{L} B \Longleftrightarrow \mu_{A}(x) \leq_{L} \mu_{B}(x) \text { for every } x \in X .
$$

\section{Unbalanced interval-valued OWA operators}

In the literature, interval-valued OWA operators are usually defined using one single numerical value for each weight. However, in this work we go one step further and propose the possibility of using two different values, one for the upper bounds and another for the lower bounds. For this reason, we propose the following definition. 
Definition 7 Let $\leq_{L}$ be an admissible on $L([0,1])$. Consider two weighing vectors $\tilde{w}=\left(w_{1}, \ldots, w_{n}\right), \tilde{v}=\left(v_{1}, \ldots, v_{n}\right) \in[0,1]^{n}$ such that $w_{1}+\ldots+w_{n}=1$ and $v_{1}+\ldots+v_{n}=1$, with $\tilde{v} \neq \tilde{w}$. An Unbalanced Interval-Valued OWA operator (UIVOWA) is a mapping

$$
\operatorname{UIVOW} A_{\left[\tilde{w}, \tilde{v}, \leq_{L}\right]}:(L([0,1]))^{n} \longrightarrow[0,1]^{2}
$$

defined by

$$
\left.\operatorname{UIVOW} A_{[\tilde{w}, \tilde{v}, \leq]}\left(\left[\underline{X}_{1}, \bar{X}_{1}\right], \ldots,\left[\underline{X}_{n}, \bar{X}_{n}\right]\right)=\left(\sum_{i=1}^{n} w_{i} \underline{X}_{(i)}, \sum_{i=1}^{n} v_{i} \bar{X}_{(i)}\right)\right)
$$

where $\left[\underline{X}_{(n)}, \bar{X}_{(n)}\right] \leq_{L} \ldots \leq_{L}\left[\underline{X}_{(1)}, \bar{X}_{(1)}\right]$.

Remark 4 OWA operators in the unit hypercube are particular instances of aggregation functions. Furthermore, ote that if we let $\tilde{v}=\tilde{w}$ then we recover the usual definition of interval-valued OWA operator. However, Definition 7 does not in principle correspond to an interval-valued aggregation function, since, to start with, it does not provide an interval as its result. To see it, consider, for instance, the vectors of weights

$$
\tilde{w}=(1,0) \text { and } \tilde{v}=(0,1)
$$

Then, if we consider the lexicographical order with respect to the first component and we take the intervals $[0,0]$ and $[0.8,1]$. Then we have that $[0,0] \leq_{l e x 1}$ $[0.8,1]$ and we arrive at

$$
U \operatorname{IVOW} A([0.8,1],[0,0])=(0.8,0)
$$

which is not an interval.

We intend now to study the conditions which allow us to state that UIVOWA operators are in fact interval-valued aggregation functions. First of all, we have the following trivial result, whose proof is straight.

Proposition 3 For every admissible order $\leq_{L}$ and for every pair of weighting vectors $\tilde{w}, \tilde{v} \in[0,1]^{n}$ it holds that

1. $U I V O W A_{\left[\tilde{w}, \tilde{v}, \leq_{L}\right]}([1,1], \ldots,[1,1])=(1,1)$;

2. $U I V O W A_{\left[\tilde{w}, \tilde{v}, \leq_{L}\right]}([0,0], \ldots,[0,0])=(0,0)$.

So in order to recover an interval-valued aggregation function we only need to consider two points:

- Which conditions ensure that the function UIVOWA is monotone with respect to the considered order $\leq_{L}$, and

- which conditions ensure that $\sum_{i=1}^{n} w_{i} \underline{X}_{(i)} \leq \sum_{i=1}^{n} v_{i} \bar{X}_{(i)}$; i.e., when we recover an interval.

None of these questions is trivial. Regarding the second one, for instance, we have the following result. 
Proposition 4 Let be $\leq_{L}$ be an admissible order. Let $\tilde{v}, \tilde{w} \in[0,1]^{n}$ be two weighing vectors such that it exists $j_{0} \in\{1, \ldots, n\}$ with $w_{i}=v_{i}$ for every $i<j_{0}$ and $w_{j_{0}}>v_{j_{0}}$. Then, UIVOW $A_{\left[\tilde{w}, \tilde{v}, \leq_{L}\right]}$ does not provide an interval as its result.

Proof. First of all, it is clear that

$$
\sum_{k=1}^{j_{0}-1} w_{k}=\sum_{k=1}^{j_{0}-1} v_{k}
$$

and consequently

$$
\sum_{k=j_{0}}^{n} w_{k}=\sum_{k=j_{0}}^{n} v_{k}
$$

We are going to prove that whatever the weighting vectors are, we can find $n$ intervals such that the image of the corresponding UIVOWA operator is not an interval.

We have that

$$
\left.\operatorname{UIVOW} A_{\left[\tilde{w}, \tilde{v}, \leq_{L}\right]}\left(\left[\underline{X}_{1}, \bar{X}_{1}\right], \ldots,\left[\underline{X}_{n}, \bar{X}_{n}\right]\right)=\left(\sum_{i=1}^{n} w_{i} \underline{X}_{(i)}, \sum_{i=1}^{n} v_{i} \bar{X}_{(i)}\right)\right)
$$

The result is an interval if and only if

$$
\sum_{i=1}^{n} w_{i} \underline{X}_{(i)} \leq \sum_{i=1}^{n} v_{i} \bar{X}_{(i)}
$$

Let's consider the following intervals:

i.) $\mathbf{x}_{\mathbf{i}}=[1,1]$ for $0 \leq i \leq j_{0}$.

ii.) $\mathbf{x}_{\mathbf{i}}=[0,0]$ for $j_{0}+1 \leq i \leq n$.

Observe that the $n$-tuple $\left(\mathbf{x}_{\mathbf{1}}, \ldots, \mathbf{x}_{\mathbf{n}}\right)$ is already ordered in decreasing order. Taking into account the intervals that we have chosen, Eq.(4) is the same as

$$
\sum_{i=1}^{j_{0}} w_{i} \leq \sum_{i=i}^{j_{0}} v_{i}
$$

This is equivalent to

$$
\sum_{i=1}^{j_{0}-1} w_{i}+w_{j_{0}} \leq \sum_{i=i}^{j_{0}-1} v_{i}+v_{j_{0}}
$$

But the first $j_{0}-1$ weights are the same, so actually we arrive at $w_{j_{0}} \leq v_{j_{0}}$, which contradicts the definition of $j_{0}$.

In fact, the following result is also straight. 
Proposition 5 Let $\leq_{L}$ be an admissible order in $L([0,1])$ and let $\tilde{w}, \tilde{v} \in[0,1]^{n}$ be weighting vectors such that $w_{i} \leq v_{i}$ for every $i \in\{1, \ldots, n\}$. Then, for every $\left(\left[\underline{X}_{1}, \bar{X}_{1}\right], \ldots,\left[\underline{X}_{n}, \bar{X}_{n}\right]\right) \in(L([0,1]))^{n}$ it holds that

$$
\sum_{i=1}^{n} w_{i} \underline{X}_{(i)} \leq \sum_{i=1}^{n} v_{i} \bar{X}_{(i)} .
$$

Proof. It is a straight consequence of the monotonicity of OWA operators.

Note that, since usual OWA operators are aggregation functions, it follows that $\sum_{i=1}^{n} v_{i}, \bar{X}_{(i)} \leq 1$, so, once the output is an interval, it is in fact an element of $L([0,1])$.

Besides, getting conditions for every possible admissible order is a very complicate work. For this reason, we only focus in this work in the case of lexicographical orders. As a first result, we can state the following.

Example 3 Let $\leq_{l e x 1}$ be the lexicographical order with respect to the first component. Consider the weighting vectors $w=(0,1)$ and $v=(0.5,0.5)$. Then

$\operatorname{UIVOW} A_{\left[\tilde{w}, \tilde{v}, \leq_{l e x 1}\right]}([0.9,0.9],[0,0])=(0.9 \cdot 0+0 \cdot 1,0.9 \cdot 0.5+0 \cdot 0.5)=$ $(0,0.45)$.

Similarly, UIVOW $A_{\left[\tilde{w}, \tilde{v}, \leq_{l e x 1}\right]}([0.8,1],[0,0])=(0.8 \cdot 0+0 \cdot 1,1 \cdot 0.5+0 \cdot 0.5)=$ $(0,0.5)$. We have that $[0.8,1] \leq_{\text {lex } 1}[0.9,0.9]$ but $[0,0.5) \geq_{\text {lex } 1}(0,0.45)$ (seen as intervals), so the operator UIVOWA is not monotonic in this case.

3.1 The problem of recovering an interval

Let's discuss now when we can ensure that we recover an interval from an UIVOWA operator. Our interest lies in studying when the inequality

$$
\sum_{i=1}^{n} w_{i} \underline{X}_{(i)} \leq \sum_{i=1}^{n} v_{i} \bar{X}_{(i)}
$$

holds. Note that if the considered intervals are degenerate, i.e., if $\underline{X}_{i}=\bar{X}_{i}$ for every $i \in\{1, \ldots, n\}$, then the inequality is equivalent to:

$$
\sum_{i=1}^{n}\left(w_{i}-v_{i}\right) \underline{X}_{(i)} \leq 0 .
$$

In this situation, the considered interval-valued fuzzy sets actually correspond to fuzzy sets. In this setting, in general, we only recover a degenerate interval for every possible choice of the input degenerate intervals if the weighing vectors $\tilde{w}$ and $\tilde{v}$ are the same. But in this case, we fall into the usual (real-valued) definition of OWA operator.

Besides, note that since the class of admissible order is so large, it is far out from the scope of the present work to provide a full characterization result. For this reason, we are going to focus in the case of lexicographical orders and we leave for future works a full analysis.

In order to get a characterization result, we start with the following lemma. 
Lemma 1 Let $\mathbf{x}, \mathbf{y} \in[0,1]^{n}$ be two weighting vectors. Then the following statements are equivalent.

1. $\sum_{j=1}^{i} x_{j} \leq \sum_{j=1}^{i} y_{j}$ for all $i=1, \ldots, n$.

2. $\sum_{i=1}^{n} x_{i} t_{i} \leq \sum_{i=1}^{n} y_{i} t_{i}$ for all $t_{i} \in[0,1]$ such that $t_{1} \geq t_{2} \geq \ldots \geq t_{n} \geq 0$.

Proof. Let's start proving that 1 . implies 2 . From our hypothesis, since $x_{1} \leq$ $y_{1}$, it follows that for every $a_{1} \geq 0$, the inequality

$$
a_{1} x_{1} \leq a_{1} y_{1}
$$

holds. In the same way, $x_{1}+x_{2} \leq y_{1}+y_{2}$, so for $a_{2} \geq 0$ it holds that

$$
a_{2}\left(x_{1}+x_{2}\right) \leq a_{2}\left(y_{1}+y_{2}\right)
$$

In this way, for each $i \in\{1, \ldots, n\}$ we get an inequality

$$
a_{i}\left(x_{1}+\cdots+x_{i}\right) \leq a_{i}\left(y_{1}+\cdots+y_{i}\right)
$$

for any $a_{i} \geq 0$. If we add all these inequalities up, we arrive at

$$
\begin{aligned}
& \left(a_{1}+\ldots+a_{n}\right) x_{1}+\left(a_{2}+\ldots+a_{n}\right) x_{2}+\ldots+a_{n} x_{n} \\
& \leq\left(a_{1}+\ldots+a_{n}\right) y_{1}+\left(a_{2}+\ldots+a_{n}\right) y_{2}+\ldots+a_{n} y_{n}
\end{aligned}
$$

for all $a_{1}, \ldots, a_{n} \geq 0$. If we define now $t_{1}=\left(a_{1}+\ldots+a_{n}\right), t_{2}=\left(a_{2}+\ldots+\right.$ $\left.a_{n}\right), \ldots, t_{n}=a_{n}$, we see that (2) holds.

The fact that (2) implies (1) is trivial, since it is enough to take $t_{1}=t_{2}=$ $\ldots=t_{i}=1$ and $t_{i+1}=t_{i+2}=\ldots=t_{n}=0$.

Using Lemma 1, we can provide the following characterization result for UIVOW A operators associated with the lexicographical order with respect to the first variable.

Theorem 1 Let $\tilde{w}, \tilde{v} \in(0,1]^{n}$ be two weighting vectors. Then the following statements are equivalent

1. The result of UIVOW A operators associated with $\tilde{w}, \tilde{v}$ and the order $\leq_{l e x 1}$ is an interval.

2. $\sum_{i=1}^{n} w_{i} t_{i} \leq \sum_{i=1}^{n} v_{i} t_{i}$ for all $t_{i} \in[0,1]$ such that $t_{1} \geq t_{2} \geq \ldots \geq t_{n} \geq 0$.

Proof. Let us show that 1. implies 2. Suppose that the result of $U I V O W A$ is an interval. Then, in particular, the inequality (6)

$$
\sum_{i=1}^{n}\left(w_{i}-v_{i}\right) \underline{X}_{(i)} \leq 0 \text {. }
$$


must hold for every choice of $\underline{X}_{i} \in[0,1](i \in\{1, \ldots, n\})$. Since we are dealing with an admissible order, we have that $\underline{X}_{(1)} \geq \ldots \geq \underline{X}_{(n)}$, so we only need to take $t_{i}=\underline{X}_{(i)}$ to get the result.

Let us assume now that 2 . holds. Let's take $n$ intervals $\left[\underline{X}_{i}, \bar{X}_{i}\right]$ with $i \in$ $\{1, \ldots, n\}$. From the definition of UIVOWA operator, we have that

$$
\operatorname{UIVOW} A_{\left[w, v, \leq_{l e x 1}\right]}\left(\left[\underline{X}_{1}, \bar{X}_{1}\right], \ldots,\left[\underline{X}_{n}, \bar{X}_{n}\right]\right)=\left(\sum_{i=1}^{n} w_{i} \underline{X}_{(i)}, \sum_{i=1}^{n} v_{i} \bar{X}_{(i)}\right)
$$

where the chain of inequalities

$$
\underline{X}_{(1)} \geq \underline{X}_{(2)} \geq \ldots \geq \underline{X}_{(n)}
$$

must hold, since we are considering the lexicographical order with respect to the first variable, $\leq_{l e x 1}$.

Now, if we take $t_{i}=\underline{X}_{(i)}$, from (2) we have that

$$
\sum_{i=1}^{n} w_{i} \underline{X}_{(i)} \leq \sum_{i=1}^{n} v_{i} \underline{X}_{(i)}
$$

But, as we are dealing with intervals, $\underline{X}_{(i)} \leq \bar{X}_{(i)}$, so the previous inequality implies that

$$
\sum_{i=1}^{n} w_{i} \underline{X}_{(i)} \leq \sum_{i=1}^{n} v_{i} \bar{X}_{(i)}
$$

and the result holds.

Corollary 1 Let $\tilde{w}, \tilde{v} \in(0,1]^{n}$ be two weighting vectors. Then the following statements are equivalent

1. The result of UIVOW A operators associated with $\tilde{w}, \tilde{v}$ and the lexicographical order with respect to the first component is an interval.

2. For every $i \in\{1, \ldots, n\}$, it holds that

$$
\sum_{j=1}^{i} w_{j} \leq \sum_{j=1}^{i} v_{j}
$$

Proof. Straight by Lemma 1 and Theorem 1 .

We can make an analogous study for the case of the lexicographical order with respect to the second variable.

Theorem 2 Let $\tilde{w}, \tilde{v} \in(0,1]^{n}$ be weighting vectors. Then the following statements are equivalent

1. The result of UIVOW A operators associated with $\tilde{w}, \tilde{v}$ and the lexicographical order with respect to the second variable is an interval.

2. $\sum_{i=1}^{n} w_{i} t_{i} \geq \sum_{i=1}^{n} v_{i} t_{i}$ for all $t_{i} \in[0,1]$ such that $t_{n} \geq t_{n-1} \geq \ldots \geq t_{1} \geq 0$. 
Proof. Analogous to that of Theorem 1.

Corollary 2 Let $\tilde{w}, \tilde{v} \in(0,1]^{n}$ be two weighting vectors. Then the following statements are equivalent

1. The result of UIVOW A operators associated with $\tilde{w}, \tilde{v}$ and the lexicographical order with respect to the second variable is an interval.

2. $\sum_{j=1}^{i} w_{j} \leq \sum_{j=1}^{i} v_{j}$ for all $i=1, \ldots, n$

Proof. Straight from Theorem 2.

Note that we have the following result.

Proposition 6 Let $\tilde{w}, \tilde{v} \in[0,1]^{n}$ be weighting vectors. Then, the following statements are equivalent.

1. $\sum_{j=1}^{i} w_{j} \leq \sum_{j=1}^{i} v_{j}$ for $i=1, \ldots, n-1$ (the condition for $i=n$ is trivial).

2. $1+\sum_{j=1}^{i} w_{j} \leq 1+\sum_{j=1}^{i} v_{j}$ for $i=1, \ldots, n-1$.

3. $1-\sum_{j=1}^{i} v_{j} \leq 1-\sum_{j=1}^{i} w_{j}$ for $i=1, \ldots, n-1$.

4. $\sum_{j=i+1}^{n} v_{j} \leq \sum_{j=i+1}^{n} w_{j}$ for $i=1, \ldots, n-1$.

Proof. It follows from a straight calculation.

So it follows that the conditions for both lexicographical orders are in fact the same.

\section{An illustrative example}

In order to show the usefulness of our developments, we consider now an illustrative example in which we make use of our theoretical developments about UIVOWA operators.

We are going to consider a decision making problem. In such a problem we are given a set of $n$ alternatives, $\left\{A_{1}, \ldots, A_{n}\right\}$ and we must find which is the best one according to a set of criteria.

In this particular case, experts from three different consultants have been asked to provide his/her preferences on whether it is better to invest some money in one or another of four companies, $\left\{C_{1}, C_{2}, C_{3}, C_{4}\right\}$. In order to take into account uncertainty about data, each consultant has provided a final grade for the quality of the investment in each of the alternatives.

For instance, the first consultant has provided the following data:

$$
A_{1}=\{(\text { Company } 1,[0.3,0.8]),(\text { Company } 2,[0.56,0.72]) \text {, }
$$

(Company 3, $[0.6,0.8]),($ Company $4,[0.16,0.0,74])\}$

where each of the interval has been obtained providing as lower bound the worst of the valuations provided by the experts in the company and as upper bound the best of the valuations provided by the experts in the company. 
The results obtained from the other two consultants are the following.

$A_{2}=\{($ Company $1,[0.46,0.58]),($ Company $2,[0.4,0.4])$,

(Company 3, $[0.2,0.5]),($ Company $4,[0.75,0.8])\}$

$A_{3}=\{($ Company $1,[0.12,0.66]),($ Company $2,[0.26,0.42])$,

(Company $3,[0.7,0.7]),($ Company $4,[0.44,0.74])\}$

The first step in order to determine which is the best possible option for investment is to fuse the information coming from the three different consultants. Once this step has been accomplished, we will use our UIVOWA operators with respect to the lexicographical order with respect to the first component and with the weight vectors $\tilde{w}=(0.2,0.25,0.55)$ and $\tilde{v}=(0.25,0.35,0.4)$ in order to select the best alternative. Note that the choice of the lexicographical order with respect to the lower bound corresponds to considering better that solution which has obtained the best worst note, so it can be understood as a sort of pessimistic choice. The choice of the weights, in a real-world application, should be done via an appropriate experimental procedure, depending on the considered problem. Note also that, with this choice, we are in the setting of Corollary 1.

The results are

$$
\begin{aligned}
& \operatorname{UIVOW} A_{\left[\tilde{w}, \tilde{v}, \leq_{l e x 1}\right]}([0.3,0.8],[0.46,0.58],[0.12,0.66])=[0.233,0.689] \\
& \operatorname{UIVOW} A_{\left[\tilde{w}, \tilde{v}, \leq_{l e x 1}\right]}([0.56,0.72],[0.4,0.4],[0.26,0.42])=[0.355,0.488] \\
& \operatorname{UIVOW} A_{\left[\tilde{w}, \tilde{v}, \leq_{l e x 1}\right]}([0.6,0.8],[0.2,0.5],[0.7,0.7])=[0.4,0.634] \\
& \operatorname{UIVOW} A_{\left[\tilde{w}, \tilde{v}, \leq_{l e x 1}\right]}([0.16,0.74],[0.75,0.8],[0.44,0.74])=[0.348,0.755] .
\end{aligned}
$$

That is, putting together the information provided by each of the consultants we arrive at

$\tilde{A}=\{($ Company 1, $[0.233,0.689]),($ Company 2, $[0.355,0.488])$,

(Company 3, [0.4, 0.634]), (Company 4, [0.348, 0.755])\}.

Now we just need to get the Company which has got the best score. In order to so, we must pick a linear order between intervals, since we should be able to compare to each other any two of the alternatives. In particular, and taking into account the way in which we have built the UIVOWA operators, it seems natural that we choose again the lexicographical order with respect to the first component. According to this admissible order, we have the following ranking:

Company 3 better than Company 2 better than Company 4 better than Company 1.

That is, the best alternative in this illustrative example would be the third one. 


\section{Conclusion}

In this paper we have introduced the notion of unbalanced interval-valued OWA operator. This operator uses two different weighting vectors, one for the lower bounds and one for the upper bounds of the considered intervals. We have analyzed when this operator, defined in terms of admissible orders, are in fact interval-valued aggregation functions in the case of the lexicographical orders.

In future works we intend to consider other admissible orders, so that our theoretical developments may be fully applied in real world applications.

Acknowledgement. Authors were supported by Project TIN2013-40765$\mathrm{P}$ of the Spanish Government.

\section{References}

1. Barrenechea E, Fernandez J, Pagola M, Chiclana F, Bustince H (2014) Construction of interval-valued fuzzy preference relations from ignorance functions and fuzzy preference relations. Application to decision making. Knowledge-Based Systems 58:33 - 44

2. Beliakov G, Pradera A, Calvo T (2007) Aggregation Functions: A Guide for Practitioners. Studies In Fuzziness and Soft Computing, Springer

3. Beliakov G, Bustince H, Calvo T (2016) A Practical Guide to Averaging Functions. Springer, Berlin, New York

4. Bustince H, Barrenechea E, Pagola M, Fernandez J (2009) Interval-valued fuzzy sets constructed from matrices: Application to edge detection. Fuzzy Sets and Systems 160(13):1819 - 1840

5. Bustince H, Calvo T, Baets BD, Fodor J, Mesiar R, Montero J, Paternain D, Pradera A (2010) A class of aggregation functions encompassing twodimensional \{OWA\} operators. Information Sciences 180(10):1977 - 1989

6. Bustince H, Fernandez J, Kolesárová A, Mesiar R (2013) Generation of linear orders for intervals by means of aggregation functions. Fuzzy Sets and Systems 220:69-77

7. Bustince H, Galar M, Bedregal B, Kolesárová A, Mesiar R (2013) A new approach to interval-valued Choquet integrals and the problem of ordering in interval-valued fuzzy set applications. IEEE Trans on Fuzzy Systems 21(6):1150-1162

8. Bustince H, Barrenechea E, Pagola M, Fernandez J, Xu Z, Bedregal B, Montero J, Hagras H, Herrera F, De Baets B (2015) A historical account of types of fuzzy sets and their relationships. IEEE Trans on Fuzzy Systems In press

9. Calvo T, Kolesárová A, Komorníková M, Mesiar R (2002) Aggregation operators: properties, classes and construction methods. In: Calvo T, Mayor 
G, Mesiar R (eds) Aggregation Operators, Studies in Fuzziness and Soft Computing, vol 97, Physica-Verlag HD, pp 3-104

10. Choquet G (1953) Theory of capacities. Annales de l Institut Fourier

11. Fodor J, Roubens M (1994) Fuzzy Preference Modelling and Multicriteria Decision Support. In Theory and Decision Library

12. Grabisch M, Marichal J, Mesiar R, Pap E (2009) Aggregation Functions. Cambridge University Press

13. Komorníková M, Mesiar R (2011) Aggregation functions on bounded partially ordered sets and their classification. Fuzzy Sets and Systems 175(1):48-56

14. Miguel LD, Bustince H, Fernandez J, Induráin E, Kolesárová A, Mesiar R (2016) Construction of admissible linear orders for interval-valued atanassov intuitionistic fuzzy sets with an application to decision making. Information Fusion 27:189 - 197

15. Sambuc R (1975) Fonctions and floues: application a l'aide au diagnostic en pathologie thyroidienne. Faculté de Médecine de Marseille

16. Sanz J, Fernandez A, Bustince H, Herrera F (2013) Ivturs: A linguistic fuzzy rule-based classification system based on a new interval-valued fuzzy reasoning method with tuning and rule selection. Fuzzy Systems, IEEE Transactions on 21(3):399-411

17. Wang Z, Klir G (1992) Fuzzy Measure Theory. Plenum, New York

18. Xu Z, Yager R (2006) Some geometric aggregation operators based on intuitionistic fuzzy sets. International Journal of General Systems 35(4):417433

19. Xu Z, Yager R (2009) Intuitionistic and interval-valued intutionistic fuzzy preference relations and their measures of similarity for the evaluation of agreement within a group. Fuzzy Optimization and Decision Making $8(2): 123-139$

20. Yager R (1988) On ordered weighted averaging aggregation operators in multicriteria decisionmaking. Systems, Man and Cybernetics, IEEE Transactions on 18(1):183-190 